\title{
Assessment by MRI of inflammation and damage in rheumatoid arthritis patients with methotrexate inadequate response receiving golimumab: results of the GO-FORWARD trial
}

\author{
Philip G Conaghan, ${ }^{1,2}$ Paul Emery, ${ }^{1,2}$ Mikkel Østergaard, ${ }^{3}$ Edward C Keystone, ${ }^{4}$ \\ Mark C Genovese, ${ }^{5}$ Elizabeth C Hsia, ${ }^{6,7}$ Weichun $\mathrm{Xu}^{6}{ }^{6}$ Mahboob U Rahman ${ }^{7,8}$
}

\begin{abstract}
- An additional supplementary table is published online only. To view this fi le please visit the journal online (http://ard. bmj.com).

${ }^{1}$ Section of Musculoskeletal Disease, Leeds Institute of Molecular Medicine, University of Leeds, Leeds, UK ${ }^{2} \mathrm{NIHR}$ Leeds Musculoskeletal Biomedical Research Unit, Leeds, UK

${ }^{3}$ Department of Rheumatology, Copenhagen University Hospital at Glostrup and Hvidovre,

Copenhagen, Denmark

${ }^{4}$ Rebecca MacDonald Centre for Arthritis and Autoimmune Diseases, Mount Sinai Hospital, University of Toronto, Toronto, Canada

${ }^{5}$ Division of Immunology and Rheumatology,Stanford University, Palo Alto, California, USA

${ }^{6}$ Centocor Research and Development, Inc., Malvern, Pennsylvania, USA

7 University of Pennsylvania School of Medicine,

Philadelphia, Pennsylvania, USA 8Pfizer, Inc., Collegeville,

Pennsylvania, USA
\end{abstract}

\section{Correspondence to}

Philip G Conaghan, Section of Musculoskeletal Disease, Leeds Institute of Molecular Medicine, University of Leeds, Chapel Allerton Hospital, Chapeltown Road, Leeds LS7 4SA, UK; p.conaghan@leeds.ac.uk

Accepted 21 June 2011 Published Online First 21 July 2011

\section{(D) UNLOCKED}

This paper is freely available online under the BMJ Journals unlocked scheme, see http:// ard.bmj.com/info/unlocked.dtl

\section{ABSTRACT \\ Objective To evaluate golimumab's effect on MRI- detected inflammation and structural damage in patients with active rheumatoid arthritis (RA) despite methotrexate (MTX).}

Methods Patients $(n=444)$ were randomly assigned to placebo plus MTX, golimumab $100 \mathrm{mg}$ plus placebo, golimumab $50 \mathrm{mg}$ plus MTX, or golimumab $100 \mathrm{mg}$ plus MTX (subcutaneous injections every 4 weeks). A subset of 240 patients participated in an MRI substudy. MRIs (1.5T + contrast enhancement) of the dominant wrist and metacarpophalangeal (MCP) joints were obtained at baseline and weeks 12 and 24. Images were scored by two independent, blinded readers for synovitis (0-9 wrist only $(n=240), 0-21$ wrist + MCP $(n=223))$, bone oedema (osteitis) (0-69) and bone erosions (0-230) using the OMERACT Rheumatoid Arthritis MRI Scoring system.

Results Significant improvements in synovitis and bone oedema (osteitis) were observed in the combined golimumab plus MTX groups versus placebo plus MTX at week $12(-1.77$ vs $-0.15, p<0.001$ wrist $+\mathrm{MCP}$ and -2.00 vs $0.19, p=0.003$, respectively) and week 24 $(-1.91$ vs $-0.38, p<0.001$ wrist $+\mathrm{MCP}$ and -1.74 vs $0.71, p=0.004$, respectively). Fewer than $10 \%$ of patients had a substantial degree of erosive progression (most showed no progression) across all treatment groups (including the control group), precluding adequate evaluation of golimumab's effect on bone erosions. Conclusion Golimumab plus MTX significantly improved MRI-detected synovitis and osteitis (prognosticators of future structural damage) versus placebo plus MTX at weeks 12 and 24. The effect of golimumab on bone erosions could not be determined by semi-quantitative scoring in these RA patients with minimal progression of bone erosions.

\section{INTRODUCTION}

Reducing inflammation and consequently inhibiting structural damage, thereby preserving patient function and quality of life, are the primary goals of rheumatoid arthritis (RA) therapy. Golimumab, a human, monoclonal antibody to tumour necrosis factor $\alpha(T N F \alpha)$ agent, has demonstrated efficacy in the treatment of established RA, including sustained improvement of clinical signs and symptoms, physical function and health-related quality of life in the GO-FORWARD study of patients with RA and inadequate response to methotrexate (MTX) therapy. ${ }^{12}$ In the analysis of GO-FORWARD radiographic data, minimal radiographic progression was observed in all treatment groups throughout the 24-week placebo-controlled period, possibly due to low levels of baseline disease activity. As a result, differences between the golimumab and placebo groups in the change in modified Sharp scores from baseline to week 24 were not statistically significant. ${ }^{3}$

While conventional radiographs remain the standard reference methods for assessing destructive skeletal changes in patients with RA, radiographs are inherently limited by the lack of ability to assess pre-erosive changes that precede damage to the osseous component of the joint, a stage of disease that had been thought to be irreversible. ${ }^{4}$ In addition to being much more sensitive in detecting joint erosions, ${ }^{5-10}$ MRI can also detect pre-erosive lesions (synovitis and osteitis). The areas of bone that appear as bone oedema or osteitis on MRI have been shown to be heavily infiltrated by inflammatory cells including osteoclasts, ${ }^{11}$ and MRIdetected synovitis and osteitis have been shown to increase the risk of developing new erosions over time as detected by either MRI or radiograph. ${ }^{12-19}$ Detection and treatment of pre-erosive lesions (synovitis and osteitis) can therefore significantly alter the course of RA.

Very few large, randomised RA trials have included MRI assessments of pre-erosive lesions. The GO-FORWARD study of golimumab in the treatment of patients with established RA therefore included an MRI substudy to evaluate the effects of this anti-TNF $\alpha$ agent on MRI-assessed RA pathology.

\section{PATIENTS AND METHODS}

The study design and patient inclusion criteria of the GO-FORWARD study have been published elsewhere. ${ }^{1}$ The overall GO-FORWARD study population consisted of patients $(n=444)$ who had active RA despite MTX treatment. Patients were to have tolerated $15 \mathrm{mg} /$ week or greater of MTX for at least 3 months before screening, with receipt of a stable MTX dose of $15 \mathrm{mg} /$ week or greater but $25 \mathrm{mg} /$ week or less during the 4 -week period immediately preceding screening. A subset of the GO-FORWARD patients from eligible and willing sites participated in an MRI substudy $(n=240)$.

The GO-FORWARD study was conducted according to the principles of the Declaration of Helsinki. As such, all patients provided written informed consent before participating in the study. 
Patients were randomly assigned to receive placebo injections plus MTX capsules (group 1), golimumab $100 \mathrm{mg}$ injections plus placebo capsules (group 2), golimumab $50 \mathrm{mg}$ injections plus MTX capsules (group 3) or golimumab $100 \mathrm{mg}$ injections plus MTX capsules (group 4). Golimumab and placebo injections were administered subcutaneously every 4 weeks. At week 16, patients with $<20 \%$ improvement in both tender and swollen joint counts entered double-blind early escape, in which patients in group 1 received golimumab $50 \mathrm{mg}$ plus MTX, patients in group 2 received golimumab $100 \mathrm{mg}$ plus MTX, and patients in group 3 received golimumab $100 \mathrm{mg}$ plus MTX. Patients in group 4 who met the criteria for early escape did not have their study medication adjusted. Patients in group 1 who did not enter early escape crossed over to golimumab $50 \mathrm{mg}$ plus MTX at week 24 . Thus, the duration of the placebo-comparator portion of the study was 24 weeks.

All patients at eligible (based on technical capabilities) and willing study sites participated in the MRI substudy. MRIs of the patient's dominant wrist and metacarpophalangeal (MCP) joints were obtained at baseline and weeks 12, 24, 52, and 104 using 1.5T MRI with contrast enhancement. The MR sequences were as follows: axial T1 fast spin echo (FSE) precontrast, coronal T1 FSE precontrast, coronal short tau inversion recovery (STIR) (or T2 fat-suppressed precontrast) and coronal T1 fat-suppressed postcontrast. Additional details pertaining to the MRI procedure are provided in an online supplementary table. Results through week 24 are presented here. Images were scored by two independent readers blinded to image time point or sequence, patient identity and treatment group. Readers scored synovitis (0-9 for wrist joint, 0-21 for wrist plus MCP joints), bone oedema (osteitis) (0-69) and bone erosions (0-230), using the Rheumatoid Arthritis MRI Scoring (RAMRIS) system. ${ }^{20}$

Randomised patients at MRI substudy sites with at least one MRI image scored by at least one reader were included in the MRI analyses (intent-to-treat population). For each patient at each visit, an MRI score was calculated as the average of each MRI score provided by the two primary independent readers. If a RAMRIS score from one of the readers was missing, then the RAMRIS score from the other reader was used. If the score was missing for both readers at any given time point, the average score was considered missing.

For patients who met the early escape criteria at week 16 in groups 1,2 or 3 , the week 12 MRI RAMRIS scores were carried forward to week 24 . The following missing data rules were applied for each RAMRIS score. (1) If the score was missing from baseline through week 12, the change score at week 12 was imputed using the median change from baseline score of all patients at week 12. (2) If the week 12 score was missing, the week 12 score was imputed using the last non-missing observation (including baseline score). (3) If the baseline RAMRIS score was missing, the change score at week 12 was imputed using the median change from baseline score of all patients at week 12 . Sensitivity analyses conducted included evaluation of change from baseline to week 24 with no imputation rules (ie, including only the 151 of the 240 (63\%) MRI substudy patients who received subcutaneous study agent through week 24 with no missed doses and no missing baseline or week 24 data for all three RAMRIS scores) and evaluation of the RAMRIS bone erosion score with linear extrapolation, which was undertaken for less than $37 \%$ of patients.

Changes from baseline to week 12 and week 24 in RAMRIS synovitis (wrist joints only in 240 patients and wrist plus MCP joints in 223 patients), bone oedema (osteitis) and bone erosion scores were compared between the treatment groups using a two-sided analysis of variance on the van der Waerden normal scores $^{21}$ at a 0.05 level of significance. The primary comparison was between the combined golimumab plus MTX groups (golimumab $50 \mathrm{mg}$ plus MTX and golimumab $100 \mathrm{mg}$ plus MTX) and the placebo plus MTX group. If the results of this comparison were statistically significant, then the individual golimumab dose groups were also compared with the placebo plus MTX group. The study, however, was not powered for these individual golimumab dose group comparisons with the placebo plus MTX group, and the sample size in each individual golimumab group in the MRI substudy may not have been large enough for reliable statistical analyses. This work, therefore, focused on comparisons for the combined golimumab plus MTX versus placebo plus MTX groups.

To assess reader reliability, images of $10 \%$ of patients were randomly selected and re-read by each of the two readers. Interreader reliability and read-reread (intrareader) reliability coefficients (IRRC and RRRC, respectively) were estimated using the week 24 RAMRIS synovitis (wrist joints), bone erosion and bone oedema scores. ${ }^{22}$

\section{RESULTS}

Of the 444 patients randomised to treatment in the GO-FORWARD study, 240 participated in the GO-FORWARD MRI substudy, including 72 patients in the placebo plus MTX, 72 in the golimumab 100 mg plus placebo, 47 in the golimumab $50 \mathrm{mg}$ plus MTX, and 49 in the golimumab $100 \mathrm{mg}$ plus MTX groups. The proportions of MRI substudy patients who met the early escape criteria (8-28\%; figure 1) were consistent with those for the overall GO-FORWARD population (11-31\%) (data not shown). ${ }^{1}$ Twenty of the 240 patients missed one or more MRI assessment visits, and all but one of the 240 patients had their MRI images scored by both readers.

Baseline demographics and disease characteristics of the MRI substudy patients were generally well balanced across the randomised treatment groups, with the exception of lower mean baseline RAMRIS synovitis (wrist plus MCP), bone erosion and bone oedema (osteitis) scores in the golimumab $100 \mathrm{mg}$ plus MTX group and a lower mean baseline $\mathrm{C}$ reactive protein (CRP) concentration in the placebo plus MTX group (table 1). Baseline characteristics were also consistent with the baseline characteristics of the overall GO-FORWARD patient population. ${ }^{1}$ Mean baseline RAMRIS bone erosion scores ranged from 22.1 to 25.5 , and mean duration of disease ranged from 8.0 to 9.5 years. Low levels of active inflammation, as evidenced by median CRP concentrations ranging from 0.6 (placebo plus MTX group) to 1.0 $\mathrm{mg} / \mathrm{dl}$ (golimumab $100 \mathrm{mg}$ plus placebo group), were observed across all randomised treatment groups (table 1).

The IRRCs (inter-reader reliability) and RRRCs (intrareader reliability) based on week 24 RAMRIS scores were 0.96 and 0.99 , respectively, for bone erosion scores; 0.89 and 0.94 , respectively, for bone oedema scores; and 0.77 and 0.84 , respectively, for synovitis.

Changes in RAMRIS scores from baseline to weeks 12 and 24 are summarised in table 2 . At week 12, significant improvements in the RAMRIS wrist plus MCP synovitis ( -1.77 vs $-0.15, p<0.001)$ and RAMRIS bone oedema ( -2.00 vs 0.19 , $\mathrm{p}=0.003$ ) scores, but not in the RAMRIS bone erosion scores, were observed in the combined golimumab plus MTX group relative to the placebo plus MTX group, respectively (table 2, figure 2). The IRRC and RRRCs for changes from baseline to week 24 were 0.80 and 0.73 , respectively, for erosion, 0.81 and 0.73 , respectively, for oedema, and 0.80 and 0.74 , respectively, for synovitis. 


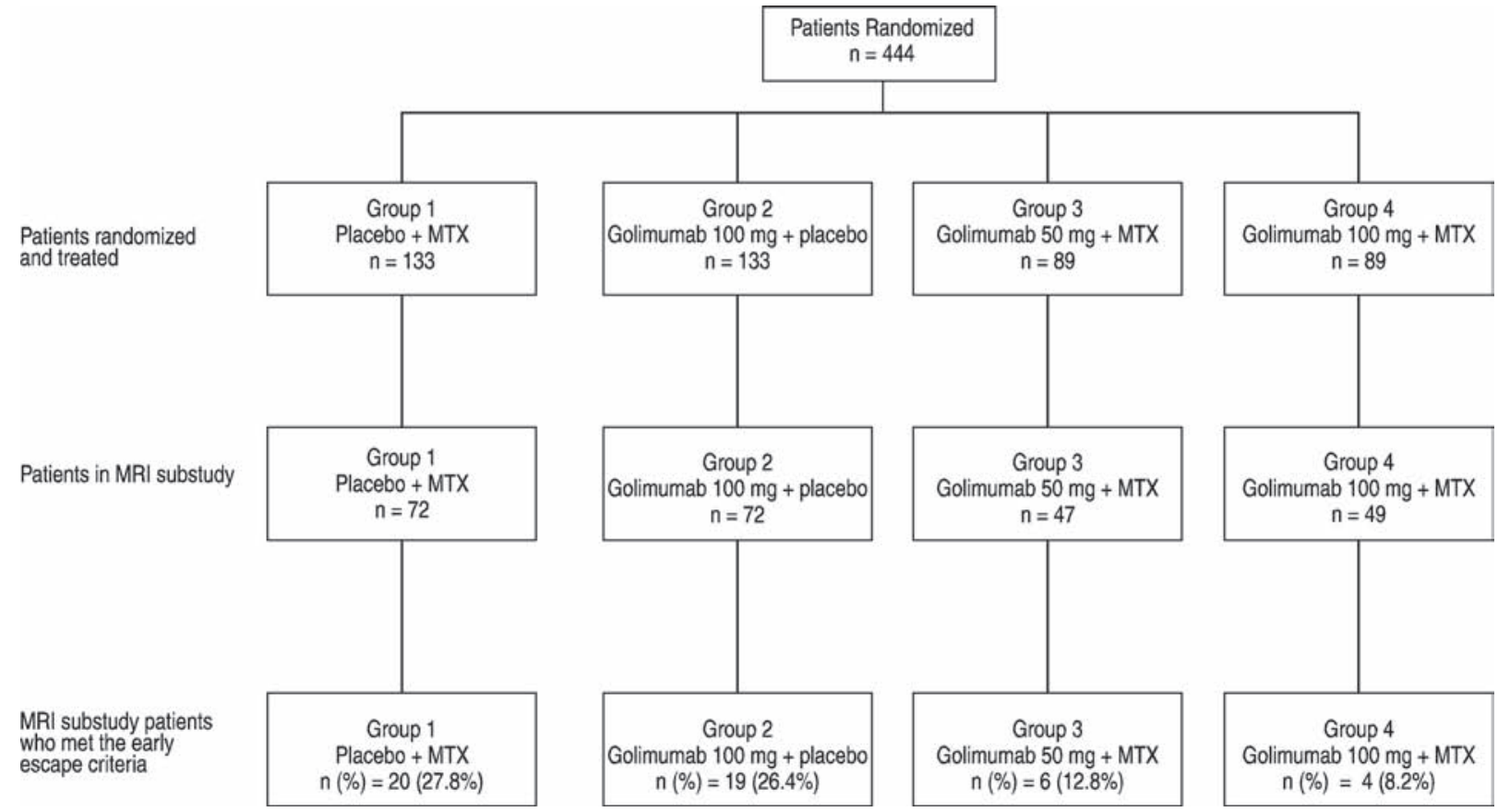

Figure 1 Patient disposition in the GO-FORWARD MRI substudy. MTX, methotrexate.

Table 1 Baseline clinical characteristics for patients in the GO-FORWARD MRI substudy

\begin{tabular}{|c|c|c|c|c|c|}
\hline \multirow[b]{2}{*}{ Characteristic } & \multirow{2}{*}{$\begin{array}{l}\text { Group } 1 \\
\text { Placebo+MTX }\end{array}$} & \multirow{2}{*}{$\begin{array}{l}\text { Group } 2 \\
\text { Golimumab } 100 \\
\text { mg+placebo }\end{array}$} & \multicolumn{3}{|l|}{ Golimumab+MTX } \\
\hline & & & Group 3: 50 mg & Group 4: $100 \mathrm{mg}$ & Groups 3 and 4 combined \\
\hline $\begin{array}{l}\text { Randomised MRI substudy } \\
\text { patients }\end{array}$ & 72 & 72 & 47 & 49 & 96 \\
\hline Women, n (\%) & $61(84.7 \%)$ & $56(77.8 \%)$ & $41(87.2 \%)$ & $42(85.7 \%)$ & $83(86.5 \%)$ \\
\hline Age (years) & $52.4 \mid 52.5$ (45.0 to 59.0$)$ & $49.2 \mid 48.5$ (41.5 to 57.5$)$ & $50.7 \mid 52.0$ (42.0 to 58.0$)$ & $49.6 \mid 49.0$ (44.0 to 53.0$)$ & $50.1 \mid 51.0(43.0$ to 57.0$)$ \\
\hline Disease duration (years) & $9.3 \mid 6.5$ (3.6 to 12.8 ) & $8.5 \mid 6.5$ (3.2 to 12.5$)$ & \begin{tabular}{l|l}
8.0 & 5.3 (2.1 to 9.8$)$
\end{tabular} & $9.5 \mid 6.8$ (2.3 to 14.5$)$ & $8.8 \mid 6.1$ (2.2 to 13.8$)$ \\
\hline $\mathrm{CRP}(\mathrm{mg} / \mathrm{dl})$ & $1.2 \mid 0.6(0.3$ to 1.9$)$ & $2.1 \mid 1.0(0.4$ to 2.6$)$ & $1.9 \mid 0.8(0.3$ to 2.0$)$ & $1.5 \mid 0.9$ (0.4 to 1.9$)$ & $1.7 \mid 0.9(0.4$ to 2.0$)$ \\
\hline $\mathrm{ESR}(\mathrm{mm} / \mathrm{h})$ & $35.3 \mid 35.5$ (19.0 to 47.0$)$ & $41.9 \mid 39.0$ (22.5 to 56.5$)$ & $41.2 \mid 38.0(22.0$ to 53.0$)$ & $39.7 \mid 34.0(25.5$ to 50.0$)$ & $40.5 \mid 36.0(23.0$ to 50.0$)$ \\
\hline vdH-S score (0-448) & $40.4 \mid 21.5$ (2.0 to 54.0$)$ & $39.1 \mid 17.3$ (3.0 to 51.0$)$ & $31.0 \mid 7.3(2.0$ to 55.0$)$ & $31.1 \mid 13.5$ (3.0 to 36.3$)$ & $31.0 \mid 13.0(2.5$ to 49.5$)$ \\
\hline \multicolumn{6}{|l|}{ RAMRIS scores } \\
\hline $\begin{array}{l}\text { Synovitis (wrist plus } \\
\text { MCP)* }(0-21)\end{array}$ & $\begin{array}{l}\mathrm{N}=56,6.7 \mid 6.8 \\
(3.3 \text { to } 9.3)\end{array}$ & $\begin{array}{l}\mathrm{N}=53,7.3 \mid 7.5 \\
(2.5 \text { to } 10.5)\end{array}$ & $\begin{array}{l}\mathrm{N}=38,7.6 \mid 7.8 \\
(4.1 \text { to } 10.5)\end{array}$ & $\begin{array}{l}N=39,6.3 \mid 6.6 \\
(3.0 \text { to } 8.5)\end{array}$ & $\begin{array}{l}N=77,7.0 \mid 7.0 \\
(4.0 \text { to } 9.5)\end{array}$ \\
\hline
\end{tabular}

Data presented are $\mathrm{n}(\%)$ or mean | median (IOR).

*Due to an inability to obtain postgadolinium images of both the wrist and the metacarpophalangeal joints at the study site, 223 patients have RAMRIS synovitis scores of the wrist plus MCP joints and 240 patients have RAMRIS synovitis scores for the wrist joint only.

CRP, C reactive protein; ESR, erythrocyte sedimentation rate; MCP, metacarpophalangeal; MTX, methotrexate; RAMRIS, Rheumatoid Arthritis MRI Scoring system.

Similar response patterns were observed at week 24, with maintenance of the significant improvements in the RAMRIS wrist plus MCP synovitis $(-1.91$ vs $-0.38, \mathrm{p}<0.001)$ and bone oedema ( -1.74 vs $0.71, p=0.004)$ scores in the combined golimumab plus MTX group relative to the placebo plus MTX group. Differences in the change from baseline to week 24 in RAMRIS bone erosion scores between the combined golimumab plus MTX and placebo plus MTX groups were not statistically significant. The percent changes from baseline to week 24 in RAMRIS synovitis (wrist plus MCP), bone oedema and bone erosion scores were $-27.0 \%,-15.8 \%$ and $+3.3 \%$, respectively, in the combined golimumab plus MTX groups and $+5.3 \%,+57.6 \%$ and $+1.0 \%$, respectively, in the placebo plus MTX group.

Results of week 24 sensitivity analyses, including an analysis based on 153/240 (64\%) patients with no missing data as well as evaluation of the RAMRIS bone erosion score with linear extrapolation (implemented for $<36 \%$ of all substudy patients), were largely supportive of the results obtained in the primary analyses of RAMRIS scores (table 2, figure 2).

MRIs from a representative patient who was randomised to receive golimumab $100 \mathrm{mg}$ plus placebo at baseline, week 12 and week 24 are shown in figure 3. Coronal STIR images 
Table 2 Summary of changes from baseline to weeks 12 and 24 in RAMRIS scores

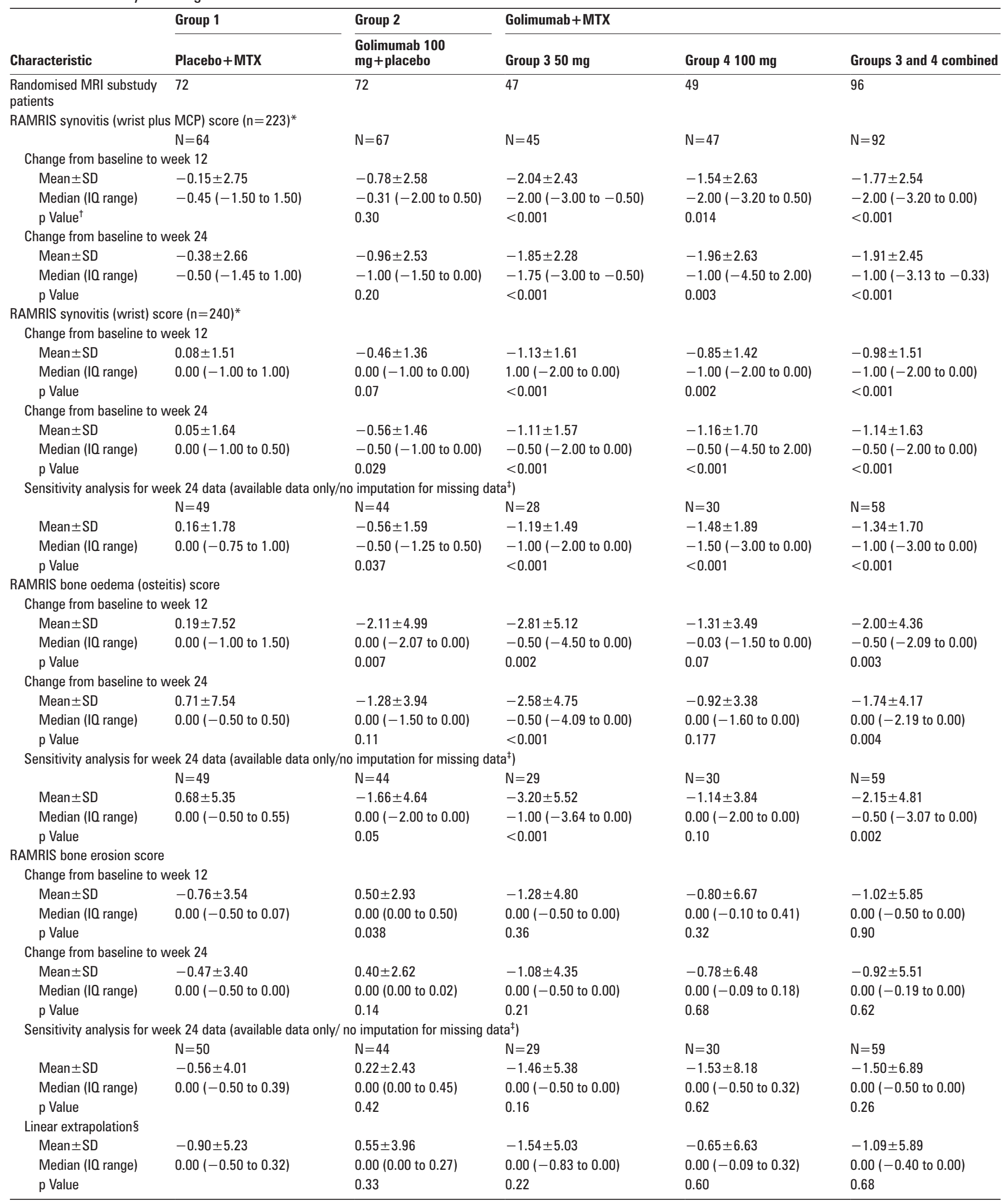

All analyses are based on the 240 randomised substudy patients unless otherwise noted in the table.

*Due to an inability to obtain postgadolinium images of both the wrist and the metacarpophalangeal joints at the study site, 223 patients have RAMRIS synovitis scores of the wrist plus MCP joints and 240 patients have RAMRIS synovitis scores for the wrist joint only.

${ }^{\dagger} A l l l p$ values derive from statistical comparisons versus group 1.

‡The sensitivity analysis employing no imputation rules included the 153/240 (64\%) substudy patients who received subcutaneous study agent through week 24 with no missed doses and no missing baseline or week 24 data.

§Evaluation of the RAMRIS bone erosion score with linear extrapolation was implemented for less than $36 \%$ of all substudy patients.

MCP, metacarpophalangeal; MTX, methotrexate; RAMRIS, Rheumatoid Arthritis MRI Scoring system. 
A.

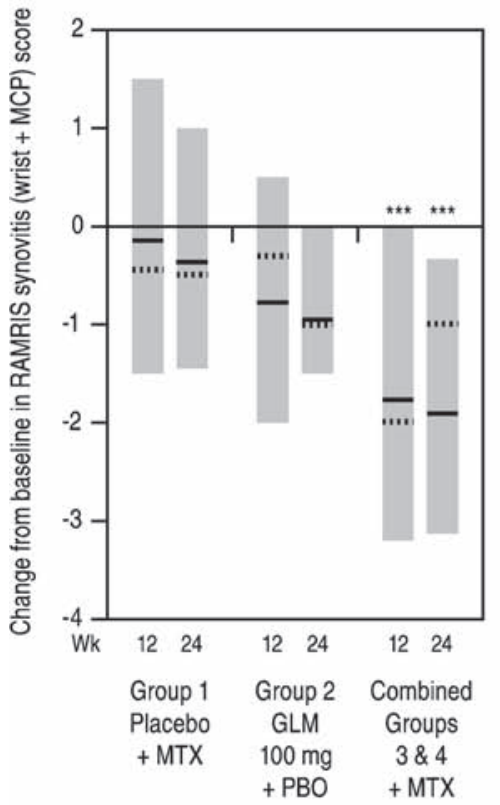

${ }^{*} p<0.05 ;{ }^{* *} p<0.01 ;{ }^{* * *} p<0.001$
B.

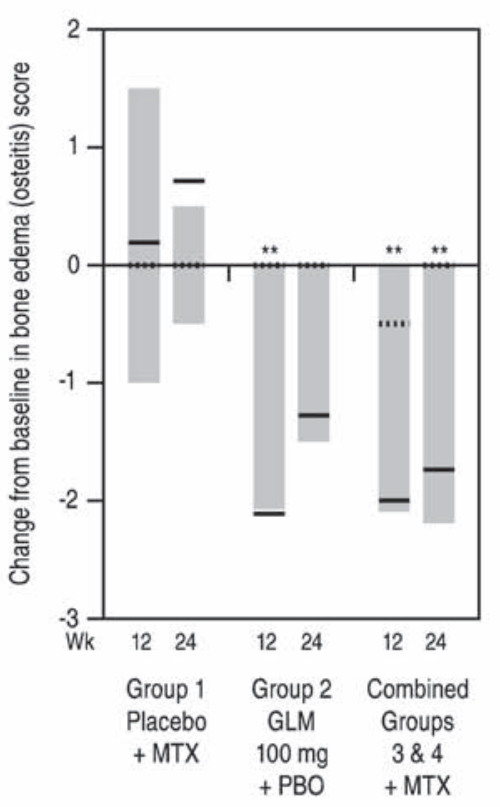

- Mean $\quad$.... Median c.

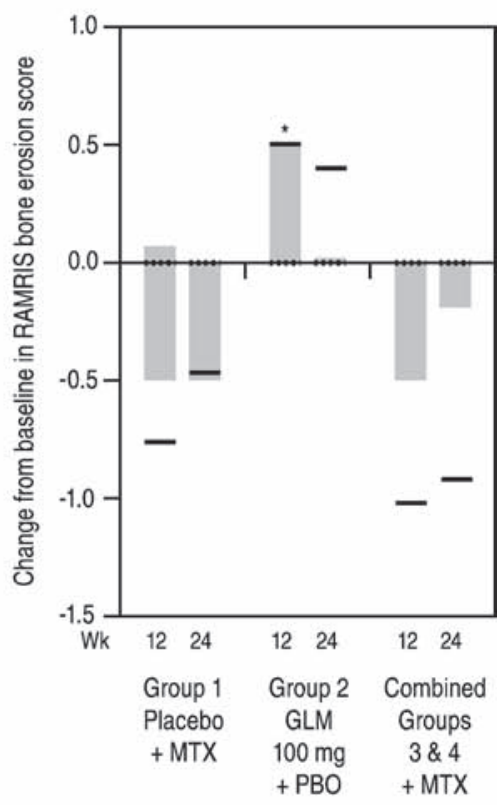

Figure 2 Changes from baseline to weeks 12 and 24 in synovitis (wrist plus metacarpophalangeal (MCP) joints (A), bone oedema (osteitis) $(B)$ and bone erosion $(C))$. Each graph shows the mean (solid horizontal line), median (dotted horizontal line) and IQR (grey bars) for changes from baseline for each treatment group. P values are for the comparison of each golimumab treatment group with placebo using analysis of variance on the van der Waerden normal scores, with ${ }^{*},{ }^{*}$ and ${ }^{* * *}$ indicating $\mathrm{p}<0.05,<0.01$ and $<0.001$, respectively. GLM, golimumab; MTX, methotrexate; PBO, placebo; RAMRIS, OMERACT Rheumatoid Arthritis MRI Score.

A. Baseline STIR

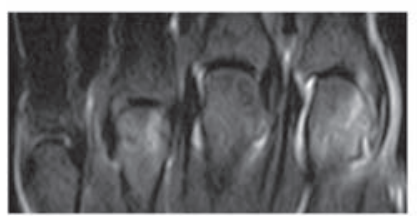

D. Baseline Post-contrast

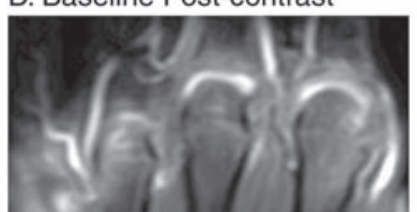

G. Baseline T1

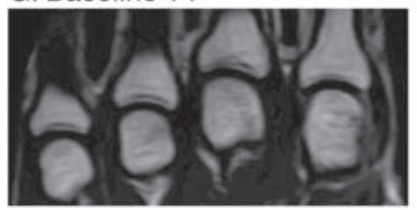

B. Week 12 STIR

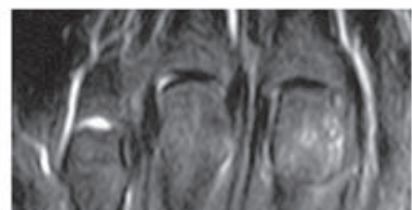

E. Week 12 Post-contrast

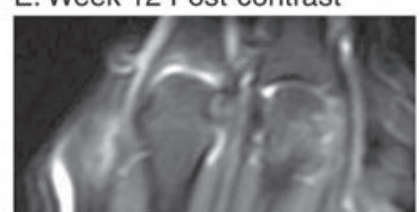

H. Week $12 \mathrm{~T} 1$

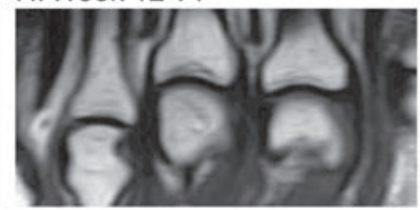

C. Week 24 STIR

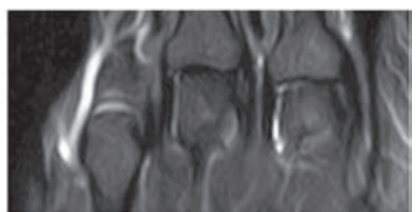

F. Week 24 Post-contrast

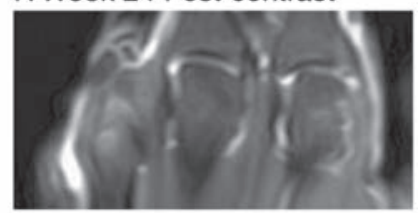

I. Week 24 T1

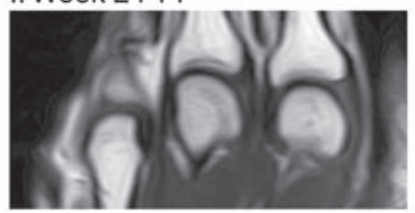

Figure $3 \mathrm{MRI}$ of the wrist at baseline $(\mathrm{A}, \mathrm{D}, \mathrm{G})$, week $12(\mathrm{~B}, \mathrm{E}, \mathrm{H})$ and week $24(\mathrm{C}, \mathrm{F}, \mathrm{I})$ of a patient randomised to receive golimumab $100 \mathrm{mg}$ plus placebo. Coronal short tau inversion recovery (STIR) images (A-C) show extensive bone oedema at baseline (A). The bone oedema has markedly decreased at week 12 (B) and has nearly resolved at week 24 (C). Corresponding postcontrast T1-weighted images with fat suppression (D-F) show substantial synovitis at baseline (D) and markedly reduced synovitis at week 12 (E) and week 24 (F). Precontrast T1-weighted images without fat suppression (G-I) show no progression of bone erosion during the 24-week follow-up period. Note: Series of consecutive images were evaluated; the images displayed here are representative but not exhaustive.

(figure $3 \mathrm{~A}-\mathrm{C}$ ) show that extensive bone oedema in many wrist bones at baseline (figure $3 \mathrm{~A}$ ) was markedly decreased at week 12 (figure 3B) and nearly resolved at week 24 (figure 3C). Corresponding postcontrast T1-weighted images with fat suppression (figure 3D-F) show substantial synovitis at baseline (figure 3D) that was markedly reduced at week 12 (figure $3 \mathrm{E}$ ) and almost resolved at week 24 (figure 3F). Precontrast T1-weighted images without fat suppression (figure 3G-I) show 
no progression of bone erosion during the 24-week follow-up period.

\section{DISCUSSION}

We evaluated a large MRI substudy of the GO-FORWARD trial, a randomised, controlled study in which the efficacy and safety of golimumab were assessed in patients with active RA despite MTX therapy. Serial measurements of synovitis, bone oedema (osteitis) and bone erosion were obtained using the RAMRIS system, which has demonstrated very good reliability and a high level of sensitivity to change. ${ }^{23} \mathrm{We}$ also observed good interreader and intrareader reliability for the pathological features scored. Results of this MRI substudy demonstrated that patients who received golimumab plus MTX had improvements in synovitis and osteitis that exceeded those observed with receipt of placebo plus MTX as early as week 12 and continuing through week 24 . Results of sensitivity analyses were largely supportive of the primary analyses.

The GO-FORWARD study results pertaining to signs and symptoms, safety and radiographic data have been reported previously. ${ }^{1-3}$ These results demonstrated that the addition of golimumab to MTX in patients with active RA despite MTX therapy significantly reduced the signs and symptoms of RA and improved physical function, while yielding a safety profile consistent with those of other anti-TNF agents. The minimal radiographic progression observed across all treatment groups through the 24 -week placebo-controlled phase of GO-FORWARD, which was likely to have been related to the low levels of baseline active inflammation (as assessed by CRP levels), precluded adequate assessment of golimumab's effect on radiographic progression in these patients with established RA. ${ }^{3}$ It has been noted that the severity of overall disease and joint damage in the population of RA patients recruited into clinical studies may be decreasing over time, ${ }^{24}$ and patients with low CRP levels are known to have less radiographic progression over time than those with high CRP levels. ${ }^{25}$ As discussed in detail by Emery and colleagues, ${ }^{3}$ it is likely that CRP, as a marker of active inflammation in RA, is a more important predictor of radiographic progression than baseline radiographic score since radiographic damage is less likely to progress if there is no active inflammation, regardless of the level of damage at baseline. For instance, RA patients with an inadequate response to MTX in the RAPID1 study of certolizumab ${ }^{26}$ had median baseline CRP levels of $\sim 1.6 \mathrm{mg} / \mathrm{dl}$, compared with $\sim 0.9 \mathrm{mg} / \mathrm{dl}$ in the current study. Despite having similar levels of radiographic damage at baseline, with modified Sharp scores of $~ 35$ in GO-FORWARD and $\sim 39$ in RAPID1, the average change in modified Sharp score (ie, progression) observed from baseline to week 24 was greater in the control group of RAPID1 (1.3) than in group 1 of GO-FORWARD (0.6). ${ }^{3} 26$ Also of note, in a different golimumab MRI substudy, conducted in MTX-naive RA patients, median baseline CRP levels were higher (1.1-1.5 mg/dl) than in the current study of MTX-experienced patients $(0.6-1.0 \mathrm{mg} / \mathrm{dl})$ and the difference between the placebo plus MTX and combined golimumab plus MTX groups for RAMRIS erosion change scores was statistically significant. ${ }^{27}$

MRI has been shown to be more sensitive to changes in bone erosions than radiographs. ${ }^{5-10}$ Thus, the expectation was that MRI would detect a difference among the GO-FORWARD treatment groups in the progression of bone erosion despite the fact that radiographic evaluation could not. ${ }^{3}$ The fact that only minimal progression of bone erosion was observed even with MRI confirms that these patients did indeed have minimal progression in structural damage during the 6-month study period. However, the fact that golimumab plus MTX has been shown to significantly improve pre-erosive inflammation, which is predictive of future bone erosion, ${ }^{12-19}$ relative to placebo plus MTX is very important, especially in light of the low levels of baseline disease activity observed in this particular patient population. All patients in the GO-FORWARD study receive golimumab treatment beyond 6 months, and it is expected that the beneficial effects of golimumab plus MTX on synovitis and bone oedema (osteitis) may prevent further progression of structural damage. While some low level MRI-documented progression of erosions was observed in terms of mean change in patients treated with golimumab $100 \mathrm{mg}$ monotherapy, it is possible that this change was within the measurement error given the range of scores for this scale.

As discussed, the RA patient population enrolled in this study actually had a low degree of active inflammation and thus minimal radiographic (erosion and joint space narrowing) progression. This may have limited evaluation of golimumab's effect on RAMRIS erosion scores. ${ }^{3}$ The limited radiographic progression observed in this study is not unique to golimumab. In an evaluation of 51 RA patients receiving adalimumab plus MTX combination therapy, no overall erosive progression or repair occurred, whereas repair of individual erosions was documented on MRI, and MRI and ultrasonography synovitis decreased. ${ }^{28}$ In a separate study, progression to radiographic erosion occurred rarely in a cohort of 50 RA patients exposed to potent disease-suppressing therapies that included TNF inhibitors, MTX and leflunomide. ${ }^{29}$ Another possible limitation to this study is that it does represent a subpopulation of the larger trial; however, the populations in each treatment arm reflected the characteristics of the full trial groups. While the responsiveness of the RAMRIS score, especially in terms of assessing erosive disease, requires further evaluation, at present this semi-quantitative score remains the standard for assessing RA MRI trials.

Overall, results of the GO-FORWARD MRI substudy have demonstrated a significant reduction in pre-erosive lesions (synovitis and osteitis) using golimumab plus MTX compared with MTX alone, even in this study population with low levels of disease activity. MRI allows measurement of inflammatory lesions such as osteitis and synovitis, as well as erosions, and thus is an important tool for objectively evaluating the effect of newer compounds on disease activity in future clinical trials enrolling patient populations with relatively lower disease activity.

Acknowledgments The authors thank the patients, investigators and study personnel who made these trials possible. The authors also thank Michelle Perate, MS of Centocor Ortho Biotech Inc., a wholly owned subsidiary of Johnson \& Johnson, Inc., who helped prepare the manuscript.

Funding This study was funded by Centocor Research and Development, Inc. and Schering Plough Research Institute, Inc.

Competing interests PGC has received consulting fees, speaking fees and/ or research grants from Astra Zeneca, Bristol-Myers Squibb, Centocor, Merck, Sharpe and Dohme, Novartis, Roche and Pfizer. PE has received consulting fees, speaking fees and/or research grants from Abbott Laboratories, Bristol-Myers Squibb, Centocor, Roche, Pfizer, UCB and Merck, Sharpe and Dohme. MCG has received grant support from, and served as a consultant to, Johnson $\&$ Johnson, Inc., New Brunswick, NJ. ECK has received consulting fees, speaking fees and/or research grants from Abbott Laboratories, Amgen Inc., AstraZeneca Pharmaceuticals LP, Bristol-Myers Squibb, Centocor, F. Hoffmann-La Roche Ltd, Genentech Inc., Novartis Pharmaceuticals Corporation, Pfizer Pharmaceuticals, Schering-Plough Corporation, UCB and Wyeth Pharmaceuticals. MØ has received consulting fees, speaking fees and/or research grants from Abbott Laboratories, Amgen Inc., Bristol-Myers Squibb, Centocor, F. Hoffmann-La Roche Ltd, Genmab, GlaxoSmithKline, Novartis Pharmaceuticals Corporation, Pfizer Pharmaceuticals, Schering-Plough Corporation, UCB and Wyeth Pharmaceuticals. ECH and WX are 
employees of Centocor, a wholly owned subsidiary of Johnson \& Johnson, Inc. (J\&J) and own stock in J\&J. MUR was formerly (during the conduct of this study) employed by Centocor R\&D Inc. Currently he is employed by Pfizer, Inc. and owns J\&J and Pfizer stocks.

Provenance and peer review Not commissioned; externally peer reviewed.

\section{REFERENCES}

1. Keystone EC, Genovese MC, Klareskog L, et al. Golimumab, a human antibody to tumour necrosis factor \{alpha\} given by monthly subcutaneous injections, in active rheumatoid arthritis despite methotrexate therapy: the GO-FORWARD Study. Ann Rheum Dis 2009;68:789-96.

2. Keystone $\mathbf{E}$, Genovese $\mathrm{MC}$, Klareskog L, et al. Golimumab in patients with active rheumatoid arthritis despite methotrexate therapy: 52-week results of the GO-FORWARD study. Ann Rheum Dis 2010:69:1129-35.

3. Emery $\mathbf{P}$, Fleischmann R, van der Heijde D, et al. The effects of golimumab on radiographic progression in rheumatoid arthritis: results of randomized controlled studies of golimumab before methotrexate therapy and golimumab after methotrexate therapy. Arthritis Rheum 2011;63:1200-10.

4. Giovagnoni A, Valeri G, Burroni E, et al. Rheumatoid arthritis: follow-up and response to treatment. Eur J Radio/ 1998;27(Suppl 1):S25-30.

5. Peterfy CG. New developments in imaging in rheumatoid arthritis. Curr Opin Rheumatol 2003;15:288-95

6. McQueen F, Lassere M, Edmonds J, et al. OMERACT Rheumatoid Arthritis Magnetic Resonance Imaging Studies. Summary of OMERACT 6 MR Imaging Module. J Rheumatol 2003;30:1387-92.

7. Hoving JL, Buchbinder R, Hall S, et al. A comparison of magnetic resonance imaging, sonography, and radiography of the hand in patients with early rheumatoid arthritis. J Rheumatol 2004;31:663-75.

8. Klarlund $\mathbf{M}$, Ostergaard M, Jensen KE, et al. Magnetic resonance imaging, radiography, and scintigraphy of the finger joints: one year follow up of patients with early arthritis. The TIRA Group. Ann Rheum Dis 2000;59:521-8.

9. Østergaard $\mathbf{M}$, Hansen $\mathbf{M}$, Stoltenberg $\mathbf{M}$, et al. New radiographic bone erosions in the wrists of patients with rheumatoid arthritis are detectable with magnetic resonance imaging a median of two years earlier. Arthritis Rheum 2003;48:2128-31.

10. Ejbjerg BJ, Vestergaard A, Jacobsen $\mathrm{S}$, et al. The smallest detectable difference and sensitivity to change of magnetic resonance imaging and radiographic scoring of structural joint damage in rheumatoid arthritis finger, wrist, and toe joints: a comparison of the OMERACT rheumatoid arthritis magnetic resonance imaging score applied to different joint combinations and the Sharp/van der Heijde radiographic score. Arthritis Rheum 2005:52:2300-6.

11. Dalbeth N, Smith T, Gray S, et al. Cellular characterisation of magnetic resonance imaging bone oedema in rheumatoid arthritis; implications for pathogenesis of erosive disease. Ann Rheum Dis 2009;68:279-82.

12. Conaghan PG, O'Connor P, McGonagle D, et al. Elucidation of the relationship between synovitis and bone damage: a randomized magnetic resonance imaging study of individual joints in patients with early rheumatoid arthritis. Arthritis Rheum 2003;48:64-71.

13. Huang J, Stewart N, Crabbe J, et al. A 1-year follow-up study of dynamic magnetic resonance imaging in early rheumatoid arthritis reveals synovitis to be increased in shared epitope-positive patients and predictive of erosions at 1 year. Rheumatology (Oxford) 2000;39:407-16

14. McQueen FM, Benton N, Perry D, et al. Bone edema scored on magnetic resonance imaging scans of the dominant carpus at presentation predicts radiographic joint damage of the hands and feet six years later in patients with rheumatoid arthritis. Arthritis Rheum 2003;48:1814-27

15. Haavardsholm EA, Bøyesen $\mathrm{P}$, Østergaard M, et al. Magnetic resonance imaging findings in 84 patients with early rheumatoid arthritis: bone marrow oedema predicts erosive progression. Ann Rheum Dis 2008:67:794-800.

16. Hetland ML, Ejbjerg B, Hørslev-Petersen $\mathrm{K}$, et al. MRI bone oedema is the strongest predictor of subsequent radiographic progression in early rheumatoid arthritis. Results from a 2-year randomised controlled trial (CIMESTRA). Ann Rheum Dis 2009;68:384-90.

17. Hetland ML, Stengaard-Pedersen K, Junker P, et al. Radiographic progression and remission rates in early rheumatoid arthritis - MRI bone oedema and anti-CCP predicted radiographic progression in the 5-year extension of the double-blind randomised CIMESTRA trial. Ann Rheum Dis 2010;69:1789-95.

18. Hodgson RJ, O'Connor P, Moots R. MRI of rheumatoid arthritis image quantitation for the assessment of disease activity, progression and response to therapy. Rheumatology (Oxford) 2008;47:13-21.

19. Bøyesen $\mathbf{P}$, Haavardsholm EA, van der Heijde D, et al. Prediction of MRI erosive progression: a comparison of modern imaging modalities in early rheumatoid arthritis patients. Ann Rheum Dis 2011;70:176-9.

20. Østergaard M, Peterfy C, Conaghan P, et al. OMERACT Rheumatoid Arthritis Magnetic Resonance Imaging Studies. Core set of MRI acquisitions, joint pathology definitions, and the OMERACT RA-MRI scoring system. J Rheumatol 2003;30:1385-6.

21. Conover WJ. Practical Nonparametric Statistics. Third Edition. Hoboken, NJ: John Wiley \& Sons, Inc 1999:396-406.

22. Vangeneugden $\mathbf{T}$, Laenen $\mathrm{A}$, Geys $\mathrm{H}$, et al. Applying concepts of generalizability theory on clinical trial data to investigate sources of variation and their impact on reliability. Biometrics 2005;61:295-304.

23. Haavardsholm EA, Ostergaard M, Ejbjerg BJ, et al. Reliability and sensitivity to change of the OMERACT rheumatoid arthritis magnetic resonance imaging score in a multireader, longitudinal setting. Arthritis Rheum 2005;52:3860-7.

24. Rahman MU, Buchanan J, Doyle MK, et al. Historical trends of patient characteristics in anti-TNF clinical trials for rheumatoid arthritis: an analysis of the literature over the past 16 years [abstract]. Ann Rheum Dis 2010;69(Suppl 3):530.

25. Smolen JS, Van Der Heijde DM, St Clair EW, et al. Predictors of joint damage in patients with early rheumatoid arthritis treated with high-dose methotrexate with or without concomitant infliximab: results from the ASPIRE trial. Arthritis Rheum 2006;54:702-10.

26. Keystone E, Heijde D, Mason D, Jr, et al. Certolizumab pegol plus methotrexate is significantly more effective than placebo plus methotrexate in active rheumatoid arthritis: findings of a fifty-two-week, phase III, multicenter randomized, double-blind, placebo-controlled, parallel-group study. Arthritis Rheum 2008:58:3319-29.

27. Ostergaard M, Emery P, Conaghan PG, et al. Golimumab and methotrexate combination therapy significantly improve synovitis, osteitis and bone erosion compared with methotrexate alone - a magnetic resonance imaging study of 318 methotrexate-naïve rheumatoid arthritis patients. Arthritis Rheum.

28. Døhn UM, Ejbjerg B, Boonen A, et al. No overall progression and occasional repair of erosions despite persistent inflammation in adalimumab-treated rheumatoid arthritis patients: results from a longitudinal comparative MRI, ultrasonography, CT and radiography study. Ann Rheum Dis 2011;70:252-8.

29. Mundwiler ML, Maranian P, Brown DH, et al. The utility of MRI in predicting radiographic erosions in the metatarsophalangeal joints of the rheumatoid foot: a prospective longitudinal cohort study. Arthritis Res Ther 2009;11:R94. 